\title{
AD-0060 - Evidence Of The Need For Diabetic Foot Health Services At Primary Health Care Clinics Around Johannesburg, Gauteng, SA
}

\author{
S. Ntuli, C. Vincent-Lambert, A . Swart. \\ Faculty of Health Sciences. University of Johannesburg
}

\section{Introduction}

Though South Africa (SA) is the only country in Africa to train podiatrists the role and need for foot health services within the public healthcare sector remains ill defined and in most cases absent. Data from the International Diabetes Federation (IDF) estimates that $7 \%$ of South Africans between the ages of 21 and 79 years have diabetes. ${ }^{1}$ Based on the 2015 population estimates for South Africa(SA), this meant that 3.85 million South Africans in this age group may have diabetes. ${ }^{2}$ It remains unclear how the foot care needs of this patient population are met as foot health services remain excluded in the primary healthcare package of services.

\section{Aim}

The aim of the study was to investigate and describe the need and role of podiatrists (foot health services) within the primary healthcare clinics in Johannesburg, SA

\section{Methods}

Two primary healthcare clinics (PHCCs) were selected to participate in the study using homogeneous sampling. Data were collected on the chief \& secondary complaint of each consenting patient presenting at the selected clinics. Data were also collected on presenting foot pathologies in all consenting patients.

\section{Results}

Data were collected from 1077 patients of which 54\% presented with a systemic condition. Diabetes was recorded in $29 \%(\mathrm{n}=314)$ of the patients.

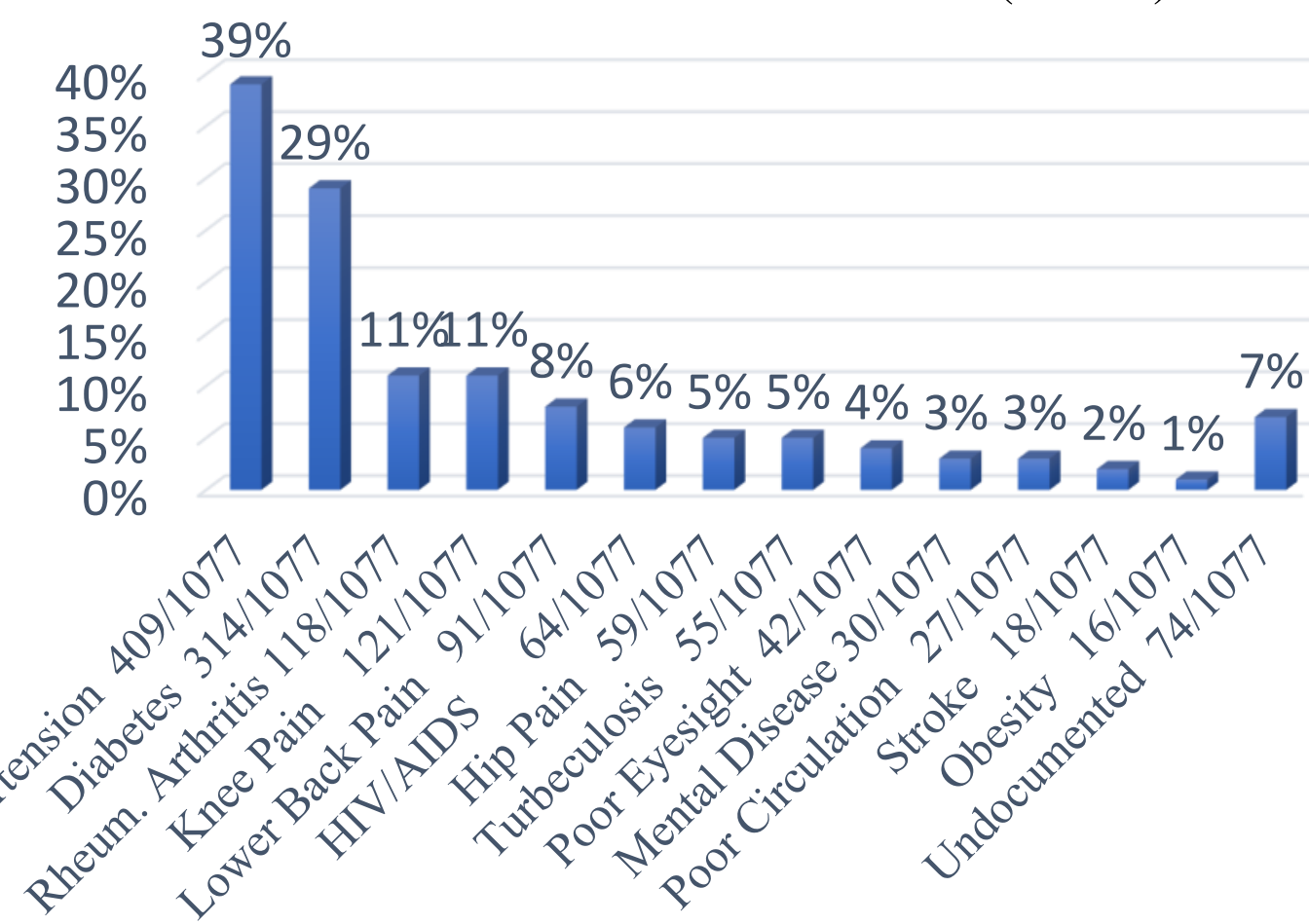

Figure 1 Presenting Systemic or Joint Disease

Diabetic neuropathy was the highest recorded diabetic foot risk factor in these patients.

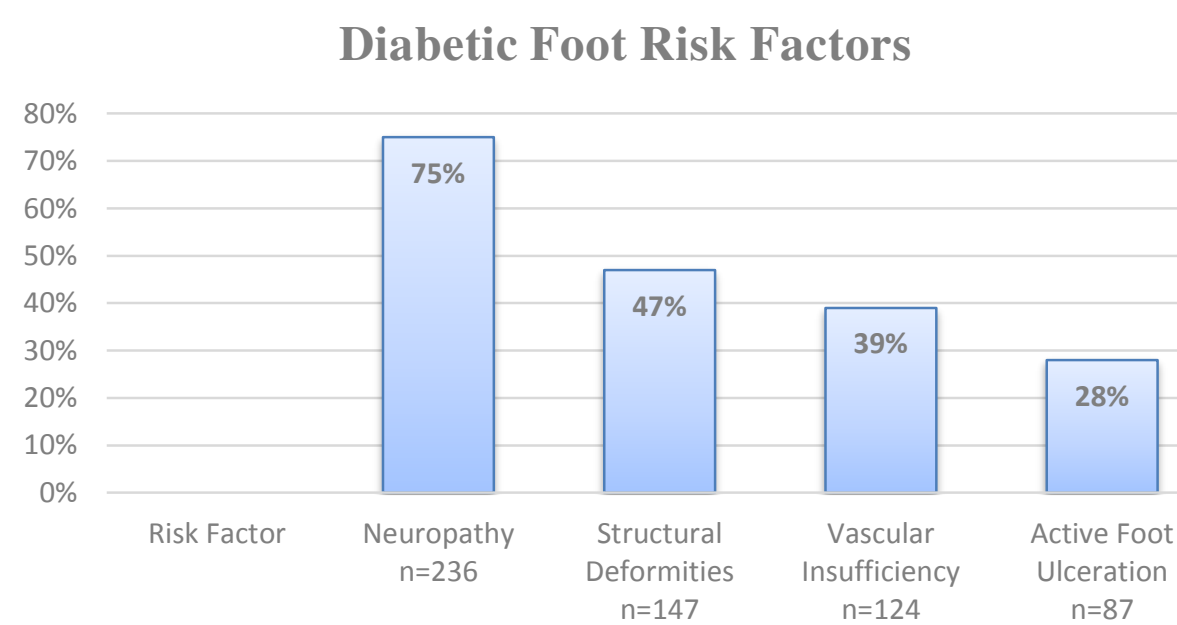

Figure 2 Diabetic Foot Risk Factors.

1. International Diabetes Federation. IDF Diabetes Atlas. Seventh Edition. Brussels, IDF. 2015.

2. Statistics South Africa. South Africa. Mid-year population estimates 2015. 2015 (July). Available from: http://www.statssa.gov.za/publications/P0302/P03022015.pdf

3. Van Rensburg et al., 2008 Primary health care in South Africa. In H. C. J. van Rensburg (Ed.), Health and health care in South Africa (pp. 412-458). Pretoria: Van Schaik Publishers.

4.GDoH, 2013 Gauteng Department of Health, Annual Report. Gauteng Provincial legislature address by the Gauteng MEC for health, Hope Papo. 2013/14
Table 1 Foot pathologies recorded in diabetic patients.

\begin{tabular}{|l|l|}
\hline Foot Pathology & Prevalence $(\%)$ \\
\hline Corns \& Calluses & $66 \%(n=207)$ \\
\hline Fissures & $32 \%(n=102)$ \\
\hline Active Ulcers & $28 \%(n=87)$ \\
\hline Infected Tinea Pedis & $25 \%(n=79)$ \\
\hline Interdigital Maceration & $21 \%(n=67)$ \\
\hline Ingrown nail & $21 \%(n=66)$ \\
\hline Numbness & $28 \%(n=89)$ \\
\hline Ischaemic feet & $22 \%(n=70)$ \\
\hline Intermittent Claudication & $17 \%(n=54)$ \\
\hline Pes Planus & $31 \%(n=98)$ \\
\hline Pes Cavus & $5 \%(n=15)$ \\
\hline Hallux valgus & $7 \%(n=22)$ \\
\hline Hammer toes & $2 \%(n=7)$ \\
\hline
\end{tabular}

\section{Discussion}

The foundation of public health delivery in SA is the primary healthcare (PHC) model. ${ }^{3}$ Thus, primary healthcare clinics (PHCCs) remain the first line of access for people needing healthcare services and in some cases the only available platform for delivery of healthcare for most of the population. The PHC model in SA is ideal for structured diabetic foot health services based at PHCCs level, as the model is premised on preventative and early risk identification. However, foot health services remain excluded from PHC package of care in SA.

In 2013, 740118 diabetic patients were treated at PHCCs across Gauteng province. ${ }^{4}$ No data is available on foot pathologies or diabetic foot risk factors recorded in these patients. This despite diabetic patients known to be at risk of diabetic foot complications. A study done on 120 diabetic patients at a district hospital in Gauteng, SA, found that $67.5 \%$ diabetic patients had never had their feet examined by either a nurse or a doctor at PHCCs. ${ }^{5}$ This may be attributed to the lack or absence of structured foot health services at PHCCs. Until this study there has been no empirical data on the prevalence of foot related pathologies in diabetic patients presenting at PHCCs in across Gauteng province.

The paucity of data on the foot related pathologies and risk factors in diabetics presenting at PHCCs, may arguably explain the omission of foot health services as part of PHC package of care. Prior to including foot health services at PHCCs, policy makers need evidence of the need and demand for such services at PHCCs. Therefore, the findings of this study are important as they highlighting the need for foot health services in diabetic patients presenting at PHCCs. The study findings showed that diabetic patients presenting at PHCCs already have foot pathologies which may potentially lead to foot complications. Diabetic foot risk factors such as neuropathy was confirmed in $75 \%$ of these patients and $28 \%$ of them already had an active foot ulcer. ${ }^{6}$ In the absence of foot health services diabetic patients remain at high risk of diabetic foot complications.

\section{Conclusion.}

There is a need for diabetic foot health services for diabetic patients presenting at PHCCs. In the absence of structured foot health services it is unclear how diabetic patients presenting at these facilities with foot pathologies and related risk factors are being managed. Thus, the findings of this study, are indicative of both the need and demand for foot health services at primary healthcare clinics.

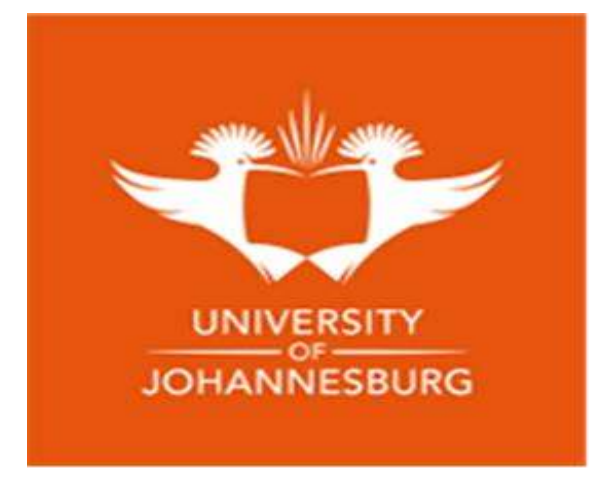

